\section{Methode zur mikrobiologischen Gewinnung der optischen Antipoden des 6-Hydroxynicotins}

\author{
M. Gloger und K. Decker
}

Biochemisches Institut an der Medizinischen Fakultät der Universität Freiburg im Breisgau

(Z. Naturforschg. 24 b, 140 [1969]; eingegangen am 4. Oktober 1968)

L- und D-6-Hydroxynicotin (1) sind die ersten Abbauprodukte des L- bzw. D-Nicotins durch das Bodenbakterium Arthrobacter oxydans ${ }^{1,2}$. Die Gewinnung der reinen optischen Antipoden von (1) ist möglich<smiles>CN1CCCC1c1ccc(O)nc1</smiles>

(1)

a) durch chemische Synthese nach Tschitschibabin ${ }^{3}$; das zunächst entstehende Racemat kann jedoch nur durch einen großen Aufwand an Zeit und Material in die Stereoisomeren aufgespalten werden. b) durch Isolierung der optischen Antipoden aus Kulturmedien des Arthrobacter oxydans; auch dieses Verfahren liefert nur geringe Ausbeuten, da sowohl die stationäre Konzentration der Substanz niedrig als auch die Reindarstellung durch störende Beiprodukte verlustreich ist. c) durch Umsetzung von D- bzw. L-Nicotin mit Enzymextrakten aus Arthrobacter oxydans nach RITTENBERG ${ }^{1}$ und DECKER ${ }^{2}$; dieses Verfahren erfordert wegen der geringen Ausbeute den Einsatz erheblicher Bakterienmengen.

Von besonderem Interesse war daher die Beobachtung, daß ein durch Anreicherungskultur auf Nicotin erhaltenes, gram-negatives Stäbchen (Stamm II) die optischen Antipoden des Nicotins in 6-Stellung zu hydroxylieren vermag und die dabei entstandenen 6-Hydroxynicotine fast quantitativ in das Medium abgibt. Im Gegensatz zu Arthrobacter oxydans bildet dieses Bakterium aus Nicotin unter den gewählten Bedingungen keinen blauen Farbstoff.

Die Hydroxylierung des D- bzw. L-Nicotins gelingt nicht nur mit wachsenden Kulturen des Stammes II, die neben Nicotin eine andere Kohlenstoffquelle (Glucose oder Glycerin) enthalten, sondern auch mit ruhenden Zellen. Das hat den Vorteil, daß Komponenten des Nährmediums die Aufarbeitung nicht stören und mit verhältnismäßig kleinen Volumina gearbeitet werden kann. Es ist möglich, die Zellen nach einem Umsatz durch Zentrifugieren aus dem Medium zu entfernen

1 L. I. Hochstein u. S. C. Rittenberg, J. biol. Chemistry 234, 156 [1959].

2 K. Decker, H. Eberwein, F. A. Gries u. M. Brühmüller, Biochem. Z. 334, 227 [1961]. und zur Hydroxylierung weiterer Ansätze zu verwenden. Unter den gewählten Versuchsbedingungen stellen die Bakterien also spezifische Hydroxylierungs-Katalysatoren dar. Der Fortgang der Hydroxylierung läßt sich durch UV-Spektroskopie des Mediums verfolgen; die Spektren des Nicotins $\left(\varepsilon_{259}=5,4\left[\mathrm{~cm}^{2} / \mu \mathrm{Mol}\right]\right)$ und des 6-Hydroxynicotins $\left(\varepsilon_{231}=12,85\left[\mathrm{~cm}^{2} / \mu \mathrm{Mol}\right], \varepsilon_{295}\right.$ $\left.=5,9\left[\mathrm{~cm}^{2} / \mu \mathrm{Mol}\right]\right)$ in $0,1 \mathrm{~N} \mathrm{HCl}$ ermöglichen eine einfache spektrophotometrische Gehaltsbestimmung ${ }^{2,4}$.

Arbeitsvorschrift: Die Bakterien des Stammes II werden auf dem folgenden Medium gezüchtet: 10,2 $\mathrm{g}$ $\mathrm{K}_{2} \mathrm{HPO}_{4}, 4,0 \mathrm{~g} \mathrm{K \textrm {K } _ { 2 }} \mathrm{PO}_{4}, 1,0 \mathrm{~g}$ Ammoniumsulfat, 3,0 g Glycerin, 1,0 g Hefeextrakt, $10 \mathrm{ml}$ einer SpurenelementLösung $\left(2,0 \mathrm{~g} \mathrm{CaCl}_{2} \cdot \mathrm{H}_{2} \mathrm{O}, 1,0 \mathrm{~g} \mathrm{MnSO}_{4} \cdot 7 \mathrm{H}_{2} \mathrm{O}, 1,0 \mathrm{~g}\right.$ $\mathrm{FeSO}_{4} \cdot 7 \mathrm{H}_{2} \mathrm{O}$ und $5,0 \mathrm{~g} \mathrm{MgSO}_{4} \cdot 7 \mathrm{H}_{2} \mathrm{O}$, suspendiert in $100 \mathrm{ml} \mathrm{0,1} \mathrm{N} \mathrm{HCl)}$; End-pH des Mediums 6,5, Wachs. tumstemperatur $31{ }^{\circ} \mathrm{C}$. Die Zellen werden nach $8-10$ Stdn. geerntet und in $0,05 \mathrm{~m}$ Phosphatpuffer suspendiert.

Zur Hydroxylierung werden ca. $2 \mathrm{~g}$ Feuchtzellen in $1 l$ einer Lösung suspendiert, die $0,05 \mathrm{M}$ Phosphatpuffer und 0,1 M L- bzw. D-Nicotin bei einem End-pH von 6,5 enthält. Die Suspension wird bei $31{ }^{\circ} \mathrm{C}$ unter Schütteln inkubiert, bis das gesamte Nicotin hydroxyliert (Spektrum) ist. Die Zellen werden abzentrifugiert und gegebenenfalls zur neuerlichen Hydroxylierung eingesetzt. Der zellfreie Überstand wird auf $\mathrm{pH} 10,5$ gebracht und zur Trockne eingeengt. Der mit $\mathrm{P}_{2} \mathrm{O}_{5}$ getrocknete Rückstand wird fein zermahlen und im S o xhlet-Apparat mit Äther erschöpfend extrahiert. Der Äther wird abgezogen und (1) dreimal aus Petroläther $\left(\right.$ Sdp. $\left.\quad 100-140^{\circ} \mathrm{C}\right)$ umkristallisiert. Der Schmelzpunkt der erhaltenen Produkte beträgt 120 bis $121^{\circ} \mathrm{C}$, die Ausbeute $80 \%$ (bezogen auf das eingesetzte Nicotin). Die UV. und IR-Spektren sind mit den früher beschriebenen ${ }^{1,2}$ identisch. Die optische Reinheit der Produkte wurde durch Bestimmung des Drehwertes zwischen 366 und $589 \mathrm{~nm}$ gesichert. In wäßriger Lösung findet man die folgenden spezifischen Drehungen :

\begin{tabular}{ccc}
\hline \multirow{2}{*}{$\lambda]$} & \multicolumn{2}{c}{$[\alpha]_{20}$} \\
& L- $(1)$ & D- $(1)$ \\
\hline $589 \mathrm{~nm}$ & $-64,4^{\circ}$ & $+62,8^{\circ}$ \\
$546 \mathrm{~nm}$ & $-77,8^{\circ}$ & $+77,3^{\circ}$ \\
$366 \mathrm{~nm}$ & $-263,9^{\circ}$ & $+259,5^{\circ}$ \\
\hline
\end{tabular}

Für die Unterstützung dieser Arbeit sind wir der Freibur ger Wissenschaftlichen Gesellschaft und der Firma Boehrin. ger, Mannheim, zu Dank verpflichtet.

3 A. E. Tschitschibabin u. A. W. Krissanow, Ber. dtsch. chem. Ges. 57, 1163 [1924].

${ }^{4}$ L. I. Hochstein u. S. C. Rittenberg, J. biol. Chemistry 234, 151 [1959]. 